\title{
New insights on the speciation history and nucleotide diversity of three boreal spruce species and a Tertiary relict
}

\author{
J Chen ${ }^{1}$, T Källman ${ }^{1}$, N Gyllenstrand ${ }^{2}$ and M Lascoux \\ Program in Evolutionary Functional Genomics, Evolutionary Biology Centre, Uppsala University, Uppsala, Sweden
}

In all, 10 nuclear loci were re-sequenced in four spruce species. Three of the species are boreal species with very large natural ranges: Picea mariana and $P$. glauca are North American, and $P$. abies, is Eurasian. The fourth species, $P$. breweriana, is a Tertiary relict from Northern California, with a very small natural range. Although the boreal species population sizes have fluctuated through the Ice Ages, $P$. breweriana is believed to have had a rather stable population size through the Quaternary. Indeed, the average Tajima's $D$ was close to zero in this species and negative in the three boreal ones. Reflecting differences in current population sizes, nucleotide diversity was an order of magnitude lower in $P$. breweriana than in the boreal species. This is in contrast to the similar and high levels of heterozygosity observed in previous studies at allozyme loci across species. As the species have very different histories and effective population sizes, selection at allozyme loci rather than demography appears to be a better explanation for this discrepancy. Parameters of Isolation-with-Migration (IM) models were also estimated for pairs of species. Shared polymorphisms were extensive and fixed polymorphisms few. Divergence times were much shorter than those previously reported. There was also evidence of historical gene flow between $P$. abies and $P$. glauca. The latter was more closely related to $P$. abies than to its sympatric relative $P$. mariana. This last result suggests that North American and Eurasian species might have been geographically much closer in the recent past than they are today.

Heredity (2010) 104, 3-14; doi:10.1038/hdy.2009.88; published online 29 July 2009

Keywords: picea; nucleotide diversity; divergence time; migration; speciation

\section{Introduction}

Classically, the history of species has been approached on two time-scales. Phylogeneticists and systematicists have focused on inferring events on deep times (millions of years) and have mostly been aiming at retrieving the topology and branch lengths of the genealogy of the group of species they were interested in. In contrast, population geneticists have been mostly interested in estimating the relative magnitude of the forces that shaped species current genetic variation: the main conceptual tool in this endeavour has been the coalescent process, which describes the genealogy of a sample of alleles taken within a given population or species. The time scale is set here by the time to the most recent common ancestor (TMRCA) of the sample. Assuming a standard coalescent the expected value of the TMRCA is equal to $4 N_{e}(1-1 / n)$, where $N_{e}$ is the effective population size and $\mathrm{n}$ is the number of sequences in the sample

Correspondence: M Lascoux, Program in Evolutionary Functional Genetics, Evolutionary Biology Centre, Uppsala University, Norbyvägen 18D, 75326 Uppsala, Sweden.

E-mail: martin.Lascoux@ebc.uu.se

${ }^{1}$ These authors contributed equally to this work.

${ }^{2}$ Current address: Department of Plant Biology and Forest Genetics, Swedish University of Agricultural Sciences, Box 7080, Uppsala 75007, Sweden.

Received 12 February 2009; revised 24 April 2009; accepted 21 May 2009; published online 29 July 2009
(Wakeley, 2008). In some cases, though certainly not in all, the effective population size and generation times are such that the TMRCA is much smaller than the time of the most recent speciation event. For example, in humans, the TMRCA for nuclear genes is on a scale of 100000 years, which is much less than the speciation time between humans and chimpanzees (c. 5-million years). However, this does not imply that the process modelled by the coalescent, namely random genetic drift, stops playing a role beyond the TMRCA. Actually, recent genomic studies have largely confirmed its importance for recent speciation events (for example, Patterson et al., 2006). More generally, Hudson and Coyne (2002) have shown that under a simple allopatric model of speciation with no selection it will take approximately 9-12 $N_{e}$ generations for the genealogies of $>95 \%$ of the loci to be reciprocally monophyletic.

This last result sheds a new light on results recently obtained in spruce species, which are going to be the object of the present investigation. By studying polymorphism and divergence at three nuclear genes among three spruce species (Picea glauca, P. mariana and P. abies) Bouillé and Bousquet (2005) observed a large number of shared polymorphisms. At first glance this may seem surprising, as the divergence between the studied species has been estimated to be around 13-20-million years ago. However, taking the upper limit of the divergence time and assuming an effective population size of 100000 and an average generation time of 50 years (Bouillé and Bousquet, 2005), 
the number of generations since divergence is a mere $4 N_{e}$, which is $<9-12 N_{e}$, and therefore shared polymorphisms are indeed expected to be common among these species, even under an allopatric model of speciation.

Shared polymorphisms can be caused by persistence in both descendant species of ancestral polymorphisms or reflect historical or current gene flow. The presence of both shared and fixed polymorphisms among spruce species means that we can take advantage of new approaches to estimate parameters of speciation, namely divergence time, effective sizes of current and ancestral population and migration rates among species. In this study we estimated nucleotide diversity at 10 nuclear loci in four spruce species and used these data to estimate parameters of speciation. The four species were the three species studied by Bouille and Bousquet (2005), namely P. glauca, P. mariana and P. abies and P. breweriana, a Tertiary relict with a limited range confined to Northern California. This is in stark contrast to P. glauca, $P$. mariana and P. abies, all of whom have continent-wide distributions: $P$. glauca and $P$. mariana are widely distributed across Canada and Alaska from the Atlantic to the Pacific Coast, while P. abies is found across Northern and Central Europe from Norway to the Urals Mountains (Figure 1). There are also other differences between $P$. breweriana and its northern relatives that make the comparison among them particularly interesting. First, although there is mounting evidence that the population sizes of $P$. abies, as well as other higher latitude species have been strongly affected by climate changes during the Quaternary (Heuertz et al., 2006; Ingvarsson, 2008; Pyhäjärvi et al., 2007), P. breweriana is believed to have had a fairly stable population size during the same period (Ledig et al., 2005 and references therein). How will this be reflected in the estimates of current and ancestral effective population sizes? Second, expected levels of heterozygosity estimated from allozyme studies in different Picea species are surprisingly stable and fall between 0.1 and 0.3 in a majority of studies (Ledig et al., 1997). For example, in comparable surveys of genetic variation of allozymes in P. abies (Lagercrantz and Ryman, 1990) and P. breweriana (Ledig et al., 2005) values of the expected heterozygosity were 0.12 in both species, even though the population sizes of the two species today differ by several orders of magnitude. Heterozygosity at allozyme loci has often been used as a proxy for effective population size (for example, Bazin et al., 2006). A strong difference in effective population sizes estimated from nucleotide diversity would put this practice into question, at least for species such as spruces. Bouillé and Bousquet (2005) dataset was very limited and they did not carry out any formal estimation of demographic parameters. For example, they used the fact that $P$. mariana and $P$. glauca do not cross naturally today, as well as the pattern of divergence in chloroplast DNA to argue that shared polymorphisms are likely to be ancestral. Although this may well be the case, caution is warranted here, as recent studies suggest that current mating pattern may not be a good proxy for ancient ones (for example, Slotte et al., 2008). Finally the presence of shared polymorphisms between $P$. abies and its North American relatives, in particular P. glauca, if confirmed on a larger dataset, could also lead to new insights on the history of spruce species. Although the species' range today is quite disjunct, the detection of large amount of spruce pollen in ice cores from Greenland indicates that boreal coniferous forest developed there some 400000 years ago during a warm interval (de Vernal and Hillaire-Marcel, 2008). Hence the different species might have been geographically closer than today and gene flow might have occurred among them.

\section{Materials and methods}

\section{Species sampling and DNA sequencing}

Between 20 and 94 individuals of each species were sampled from their natural distribution range (Supplementary File S1). Haploid DNA was extracted from individual megagametophytes using Qiagen DNeasy plant mini kit (Hilden, Germany). PCR amplifications were done using the proofreading enzyme Phusion (Finnzyme, Espoo, Finland) and successful amplifications were treated with ExoSAP-IT (USB, Cleveland, $\mathrm{OH}$, USA) before sequencing. Details regarding origin, extension times and annealing temperature as well as primer sequences for individual loci can be found in Supplementary Table S2. Sequence data were collected with

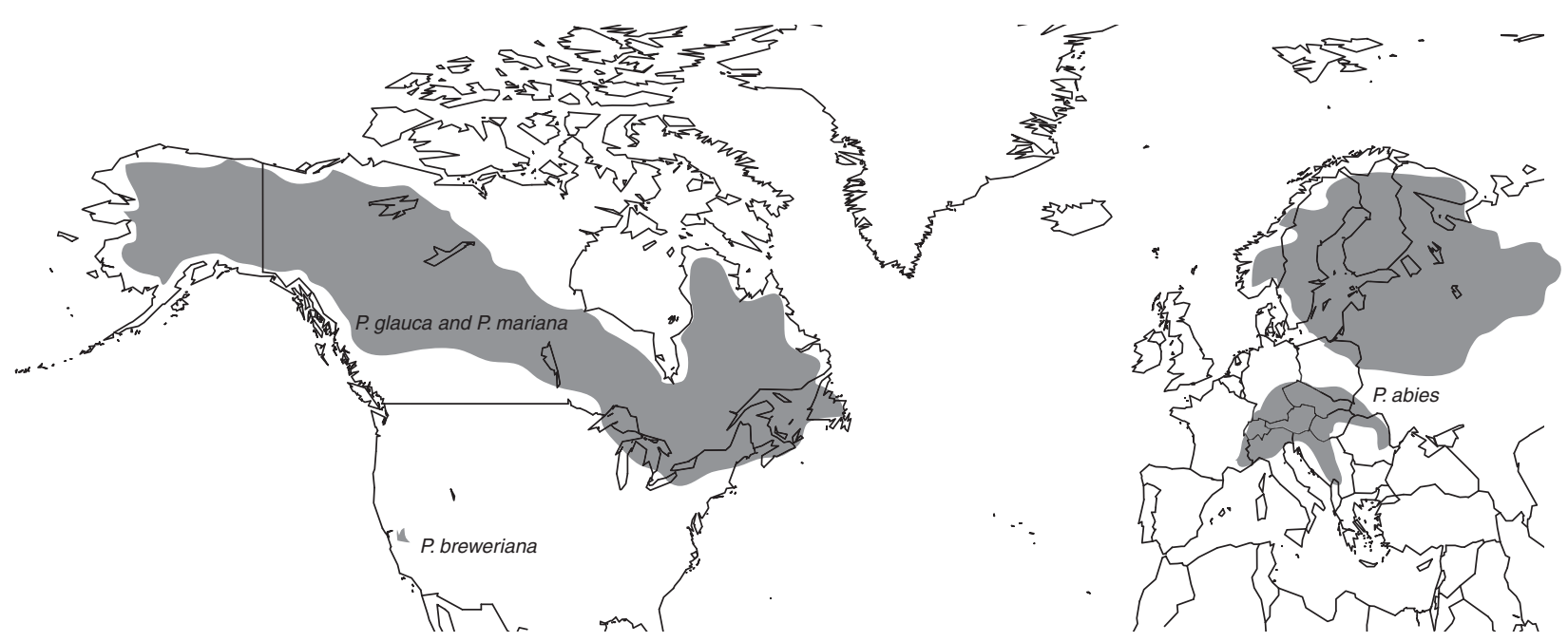

Figure 1 Natural distribution ranges of the four studied spruce species. 
either a MegaBace 1000 using ET-terminator (GE Healthcare, Piscataway, NJ, USA) or an ABI3730 with BigDye v3.1 (Applied Biosystems, Foster City, CA, USA). Sequence data were base-called, assembled and edited with the PHRED, PHRAP and CONSED software suite keeping only high quality data and, furthermore, manually checking all putative polymorphic sites (Ewing and Green, 1998; Ewing et al., 1998; Gordon et al., 1998). Sequence data for $P$. abies was partly obtained from another study on population genetics of P. abies (Heuertz et al., 2006).

\section{Sequence data analysis}

All obtained sequences were manually aligned and assigned to coding and non-coding regions by aligning genomic sequences against their corresponding mRNA sequence. Standard population genetic parameters for individual loci were calculated either in DNA sequence polymorphism (DNAsp) version 4.5 (Rozas et al., 2003) or with the programme compute (Thornton, 2003). As neutrality is one of the major assumptions of the Isolation-with-Migration (IM) model we also tested departure-from-neutrality using the Hudson-KreitmanAguadé framework and the maximum likelihood implementation of this test statistics with the programme mlHKA (Wright and Charlesworth, 2004). Recent studies have shown that the Hudson-Kreitman-Aguadé test is one of the most powerful tests to detect positive selection using polymorphism data (Innan and Kim, 2008; Zhai, Nielsen and Slatkin, 2009).

The population recombination parameter $(\rho)$ was estimated with LDhat 2.1 (McVean et al., 2002). The range of estimated $\theta$ and $\rho$ values from individual loci was later used to define previous uniform ranges in multilocus estimates of $\rho$ and $\theta$ with the software Rhothetapost (Haddrill et al., 2005).

\section{Estimation of IM parameters}

Speciation parameters were estimated under an IM model using the software MIMAR (Becquet and Przeworski, 2007). MIMAR implements a Markov Chain Monte Carlo method to estimate speciation parameters from a slightly different version of Wakeley and Hey (1997) summary statistics. Briefly, segregating sites $(S)$ are classified into four categories, $S_{1}, S_{2}, S_{\mathrm{s}}$ and $S_{\mathrm{f}}$. For each locus, $S_{1}$ and $S_{2}$ are the number of polymorphic sites unique to samples 1 and 2 ( $S_{1}$ and $S_{2}$, respectively), $S_{\mathrm{s}}$ is the number of sites with shared alleles between the two samples, and $S_{\mathrm{f}}$ is the number of sites with fixed alleles in either samples. MIMAR also differs from previous implementations of the IM model by taking recombination into account but, otherwise, relies on the same set of major assumptions. Namely, loci are assumed to be neutral, and the two species under study are more closely related than any of them is to a third species. In particular, this means that there should not be other unsampled populations exchanging genes with the sampled populations or their ancestor. Clearly this is a demanding assumption. Finally, as pointed by the authors MIMAR may not provide precise estimates unless datasets have both shared and fixed alleles between the two samples.

When assigning polymorphisms to the four categories we excluded indels and sites with missing data and used outgroup sequences to infer the derived allele frequency in the species $i, f_{i}$. Pinus taeda was the only outgroup in analyses involving P. breweriana, and both Pinus taeda and $P$. breweriana were used as outgroups (Ran et al., 2006) to minimize the error in inferring the ancestral states in pairwise analyses of the remaining three spruce species. $S_{1}, S_{2}, S_{\mathrm{s}}$ and $S_{\mathrm{f}}$ were then calculated according to the MIMAR manual supplied by Becquet and Przeworski (2007). Specifically, for polymorphic sites, if $0<f_{\mathrm{i}} \leqslant 1$ in each species, the allele is shared; if $f_{i}=0, f_{j}=1, i \neq j$, the allele is fixed in the sample $j$; and if $f_{i}=0$, and $0<f_{j}<1$, $i \neq j$, the allele is specific to sample $j$.

The IM model used in MIMAR is defined by 6 parameters: the population split time in generations, $T_{\text {geni }}$ three population mutation rates $\theta_{1}=4 N_{\mathrm{e} 1} \mu$, $\theta_{2}=4 N_{\mathrm{e} 2} \mu$ and $\theta_{\mathrm{A}}=4 N_{\mathrm{eA}} \mu$, where $N_{\mathrm{e}}$ is the effective population size of the two descendant populations and of the ancestral population and $\mu$ is the mutation rate per base pair; and the migration rates $M_{12}=4 N_{\mathrm{e} 1} m_{12}$ and $M_{21}=4 N_{\mathrm{e} 1} m_{21}$. It is to be noted that migration rates are scaled by the effective size of population 1 . The Markov Chain Monte Carlo was run at least $7 \times 10^{6}$ steps, $1 \times 10^{6}$ burning steps and sampled every 50 steps. The previous distributions and the variance of the normal kernel distributions were optimized after several test runs. This step was crucial to obtain good mixing and thereby, a good acceptance rate. The population recombination rate per base pair is defined as $\rho=4 N_{\mathrm{e}} r$, where $N_{\mathrm{e}}$ is the effective population size and $r$ is the recombination rate per base pair per generation. We ignored gene conversions, and took the average value of multilocus estimates of $\rho$ as the recombination rate between two species in the analysis. The number of Ancestral Recombination Graphs per locus was set to $X=100$.

Two demographic models were implemented. First, we used a standard neutral model in each pair of species, thereafter called the standard IM model. Second, because the average Tajima's $D$ values were negative in the three boreal species, we added population growth for those while retaining a standard neutral model for $P$. breweriana that did not show any clear evidence of departure from the standard neutral model. More complex demographic models such as bottlenecks were not considered as the MIMAR model is already extremely computer intensive and growth models gave a satisfactory fit (see below). To assess convergence and the mixing of Markov Chain Monte Carlo, we carried out two independent runs and monitored the performance of MIMAR using code provided with MIMAR (For all the parameters and model settings, please refer to Supplementary File S3).

To examine whether our models captured the main features of the history of the species studied here, we used a goodness-of-fit test. We generated simulated datasets for parameters sampled from the posterior distribution estimated by MIMAR, and compared the observed values of a number of statistics: $S_{1}, S_{2}, S_{\mathrm{s}}, S_{\mathrm{f}}, \pi, F_{\mathrm{st}}$ and Tajima's $D$ to the distribution of the summary statistics of the simulated data in accordance with Becquet and Przeworski (2007). The $P$-value for each statistics was calculated for both standard IM model and growth model.

\section{Phylogeny}

We used BEST 2.0 (Liu and Pearl, 2007), a Bayesian hierarchical model built in MrBayes (Ronquist and 
Huelsenbeck, 2003) to estimate simultaneously gene trees and the species tree. We sampled randomly one individual in each species and used Pinus taeda as an outgoup. The same 10 unlinked genes as in the MIMAR analysis were used and an inverse $\gamma$ substitution model was chosen. $100 \times 10^{6}$ steps and a total of eight chains were carried out to obtain convergence of the Markov Chain Monte Carlo. The species tree was compared with that inferred by the estimates of divergence time given by MIMAR.

\section{Results}

\section{Polymorphism}

Sequence data from 10 loci were collected from four different spruce species, three North American ones, P. breweriana, P. glauca and P. mariana and one European relative, $P$. abies. The average number of megagametophytes/species was 35. In total just over $8000 \mathrm{bp}$ of aligned sequence was obtained and 210, 26, 119 and 153 segregating sites were identified in P. abies, $P$. breweriana, $P$. glauca and $P$. mariana respectively. Estimates of standard population genetic statistics for the four different species are summarized in Figure 2 (details for individual loci are given in Supplementary Table S4). The nucleotide diversity was an order of magnitude lower in P. breweriana than in the other three species that all harboured similar levels of variation. In all four species the majority of loci showed negative Tajima's $D$ values, even though a vast majority of them were nonsignificant and for $P$. breweriana the observed values were close to zero (Figure 2). The locus Sb29 showed a deviating pattern with positive values of Tajima's $D$ in all species but $P$. breweriana. In $P$. abies and $P$. mariana all loci were polymorphic, whereas GI was monomorphic in a

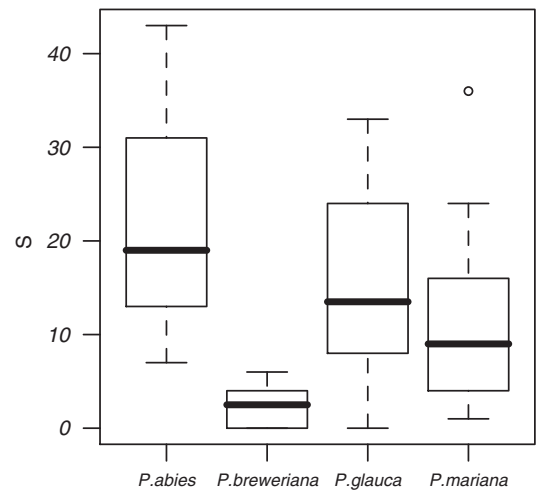

C

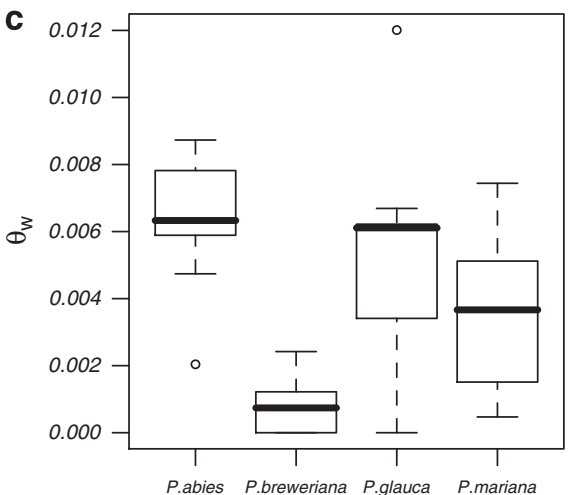

e

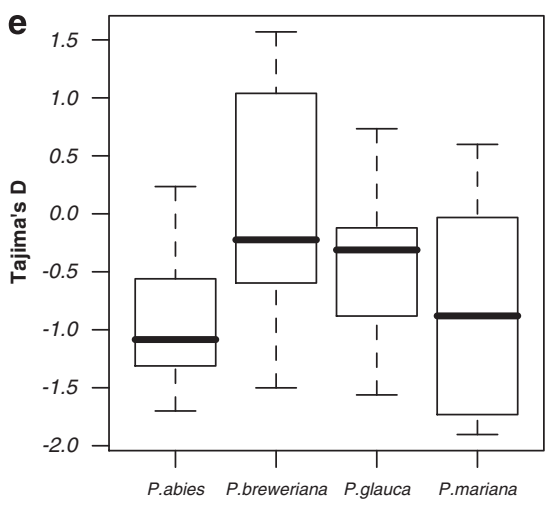

b

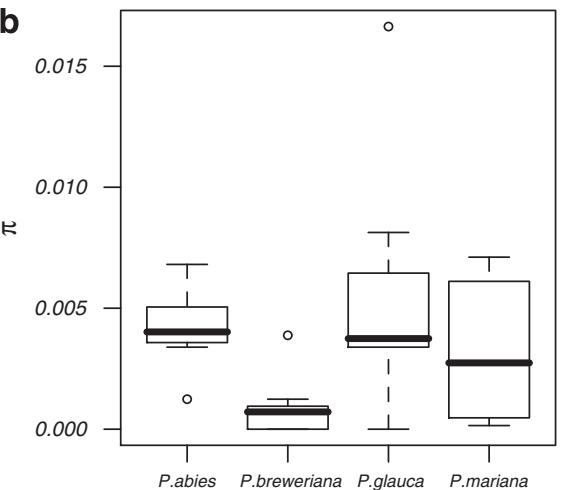

d

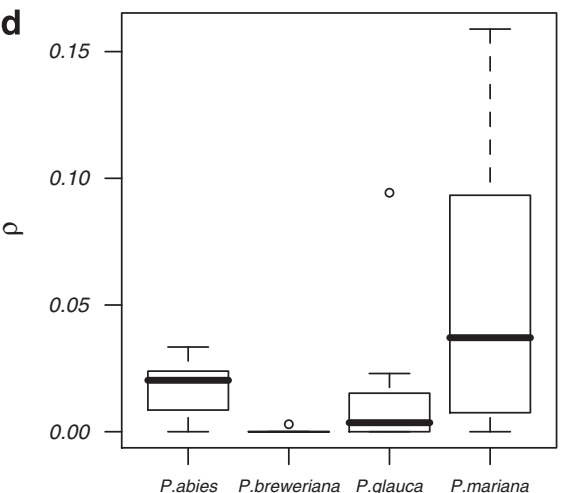

f

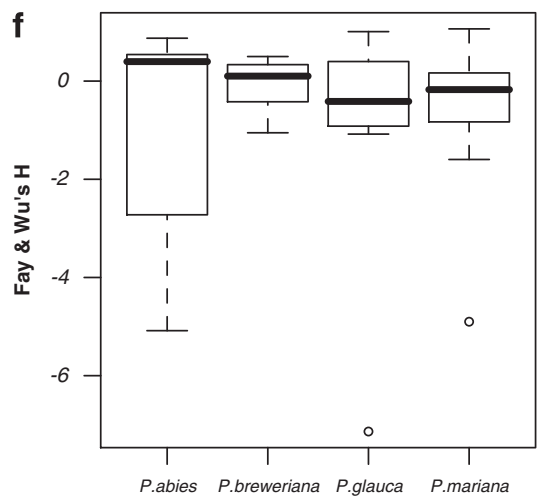

Figure 2 Polymorphism patterns among the four spruce species. Bars represent the median, boxes the interquartile range and whiskers extend out to 1.5-times the interquartile range. (a) Number of segregating sites, (b) Pairwise nucleotide diversity, (c) Watterson's $\theta$, (d) Population recombination rate $(\rho)$, (e) Tajima's D, and (f) Fay and Wu's H. 
P. glauca and loci GI, Sb16 and se1364 were fixed in $P$. breweriana. All four species had highest diversity at synonymous sites and the non-synonymous diversity was, averaged over loci, less than a third of the silent diversity (Supplementary Table S4). Finally, only ZTL deviated from neutral patterns when using multilocus Hudson-Kreitman-Aguadé tests (data not shown).

Thirty-seven indel variants between 1 and 43-bp long were detected among and within species. Only three of the indels were due to fixed length differences between species. Seven of the indel variants were found in more than one species and the remaining ones were specific to a given species. With the exception of a microsatellite shared between $P$. breweriana and $P$. mariana all identified indels in $P$. breweriana were private. $P$. glauca and $P$. abies had the highest proportions of shared indels with four indels in common. As expected most of the single nucleotide polymorphisms and indel variants were found in the non-coding regions.

\section{Speciation parameters}

The segregating sites were classified into the four categories, $S_{1}, S_{2}, S_{\mathrm{s}}$ and $S_{\mathrm{f}}$. Ten segregating sites were shared by $P$. abies, $P$. glauca and $P$. mariana. Unexpectedly, considering their current geographic distribution, $P$. abies and P. glauca shared 42 polymorphic sites, more than twice the number shared between $P$. glauca and $P$. mariana, whose distribution ranges today almost completely overlap. Three loci ZTL, COL2 and FT3 contributed most to the shared polymorphisms, whereas the fixed differences between species mainly came from FT3 and GI. It is interesting to note that in contrast to other genes, GI showed a proportionally large number of fixed differences between species, but no shared variation (Supplementary Table S3).

\section{Standard IM model}

Figures 3 and 4 show the marginal posterior distribution for parameters of the standard IM model. By assuming a mutation rate $\mu=1.0 \times 10^{-8}$ per site per generation and an average generation time $t=50$ years (Bouillé and Bousquet (2005)), we estimated the effective population size $N_{1}, N_{2}, N_{\mathrm{A}}$, the divergence time $T$ and the migration rates $M_{12}$ and $M_{21}$, in pairwise comparisons. Because of the uncertainty attached to mutation rate and generation time estimates we also provide estimates for $\mu=2.0 \times 10^{-8}$ and generation time of 25 or 50 years (Supplementary File S5).

Encouragingly, the estimates of the effective population size are consistent across the six pairwise analyses, suggesting that the standard IM model does capture some general features of the population histories (Becquet and Przeworski, 2007). P. abies has the largest effective population size estimates of the four species, around 136000-159000. Although P. glauca and $P$. mariana are largely sympatric today, the estimated effective population sizes of $P$. mariana (around 84700$97000)$ is just $2 / 3$ that of P. glauca (121 000-133000). The estimate of effective population size of $P$. breweriana was an order of magnitude smaller (11600-12900). It is interesting to note that in contrast to what has been observed in many previous studies (see Becquet, 2007 and references therein), the ancestral effective population sizes, with values ranging from 92000-125000, was smaller, or of the same order of magnitude, than the inferred effective population sizes of the three boreal species. This is in agreement with the expectations, as these populations have obviously gone through strong population growth since their inception.

The different pairwise analyses indicate that $P$. breweriana diverged first from the common ancestor of the four spruce species around 13-17 Mya and that $P$. mariana diverged from the other two boreal species around 12-15 Mya. Finally, Figure 3b shows that split of $P$. abies and P. glauca occurred nearly 8.5 Mya. Migration rates estimates were generally low, with the notable exception of the estimate of gene flow from $P$. glauca to P. abies, which had a value greater than 1 .

\section{Goodness-of-fit test for the standard IM model}

To assess the goodness-of-fit of the standard IM model we compared four summary statistics produced by coalescent simulations based on parameter values randomly sampled from the parameter posterior distributions with the corresponding values of the summary statistics from the actual data. The standard IM model gives a reasonably good fit for $S_{1}, S_{2}, S_{\mathrm{s}}$ and $S_{\mathrm{f}}, \pi$ and $F_{\mathrm{st}}$ but the fit for Tajima's $D$ tends to be poor (Figure 5, Supplementary File S6). The negative mean values of Tajima's $D$ could be explained by the presence of selection, and/or departure from constant population size. As Tajima's $D$ values were consistently negative across loci, but individual loci did not statistically depart from neutrality, a demographic explanation seems more likely (Heuertz et al., 2006).

\section{Growth model}

As Tajima's $D$ was strongly negative for the three boreal species and close to zero in $P$. breweriana, we also estimated speciation parameters using an IM model, in which the boreal species were subjected to a decrease in population size followed by population expansion and the $P$. breweriana population size was kept constant. The present effective population size estimates from the growth models were almost twice as large as the estimates obtained under the standard IM model (Figures 6 and 7 and Supplementary File S5). However, estimates of the ancestral effective population size were not affected by the change of model. In the two North American species $P$. glauca and $P$. mariana growth was initiated 2-2.6 Mya, but the latter grew much faster. $P$. abies population expansion was more recent, ca 1.852 Mya with a similar growth rate to that of $P$. mariana. The estimates of the divergence time were half of those obtained under the standard IM model and the genealogical relationships between the four species were not as clear. The species split around 6-7.8 Mya, except $P$. glauca and $P$. breweriana that diverged 13 Mya. The amount of gene flow between species pair increased, but the directions remained the same as under the standard IM model. Like in standard IM model the amount of gene flow from $P$. glauca to $P$. abies was notably high, being close to 4.4 (Supplementary File S5).

\section{Goodness-of-fit test for the growth model}

In most cases the growth model improved the goodnessof-fit for all summary statistics and in particular for Tajima's $D$, which was the one showing poorest fit under 
a
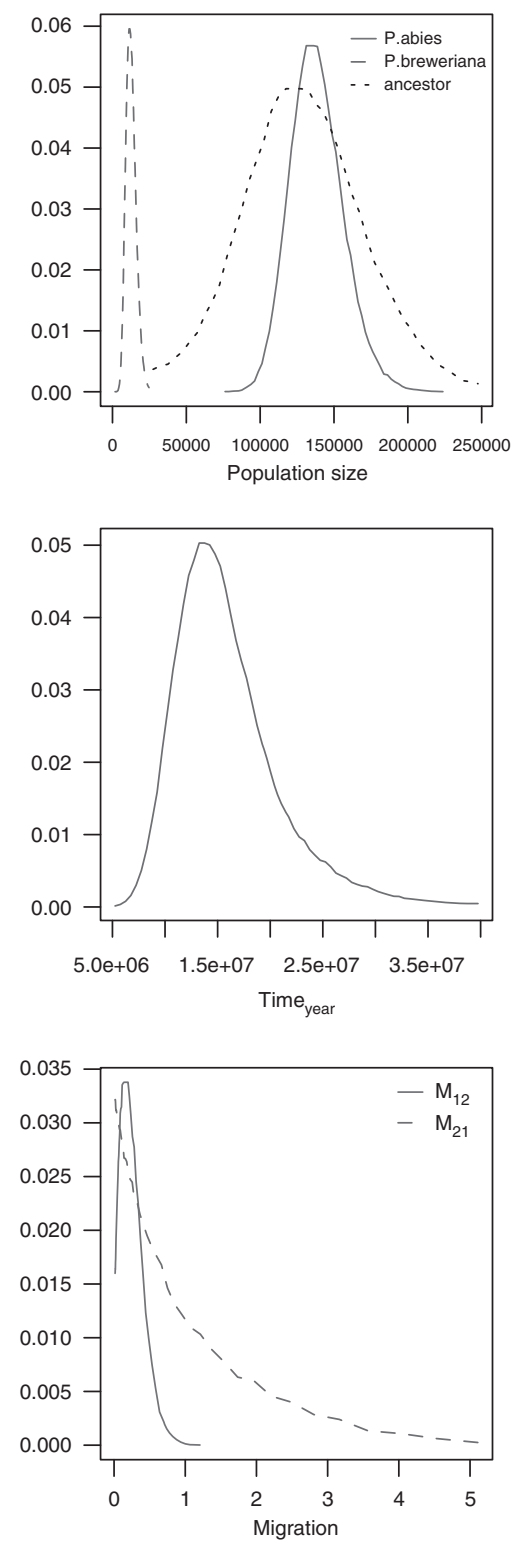

\section{b}
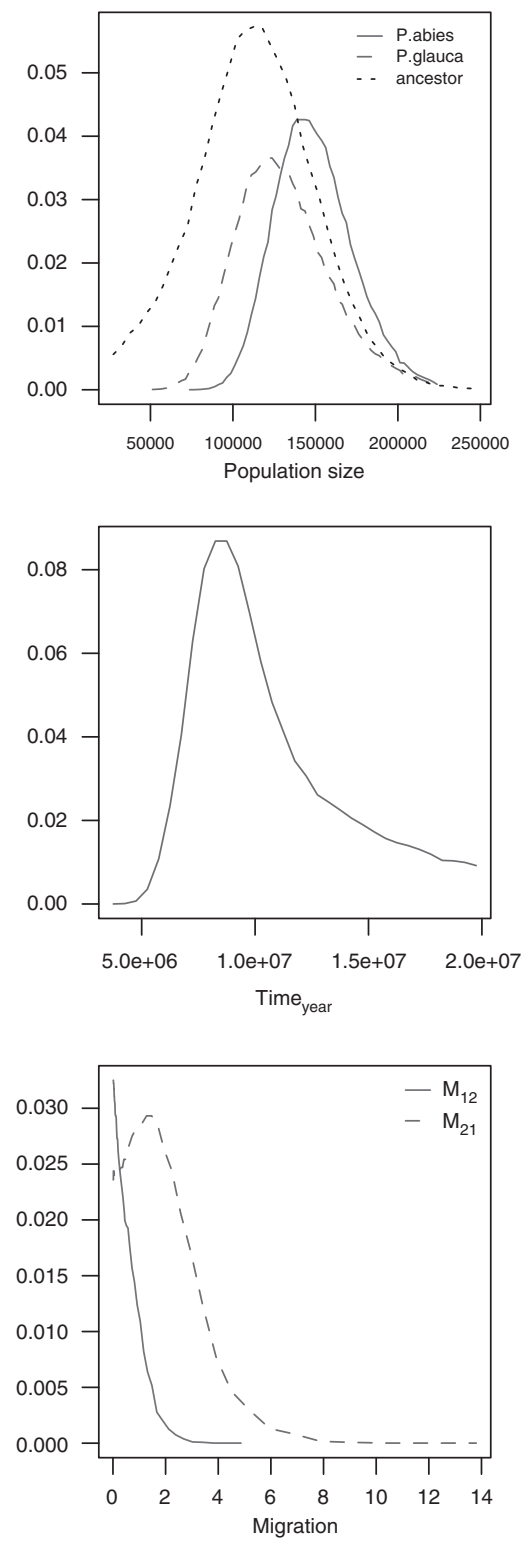

C
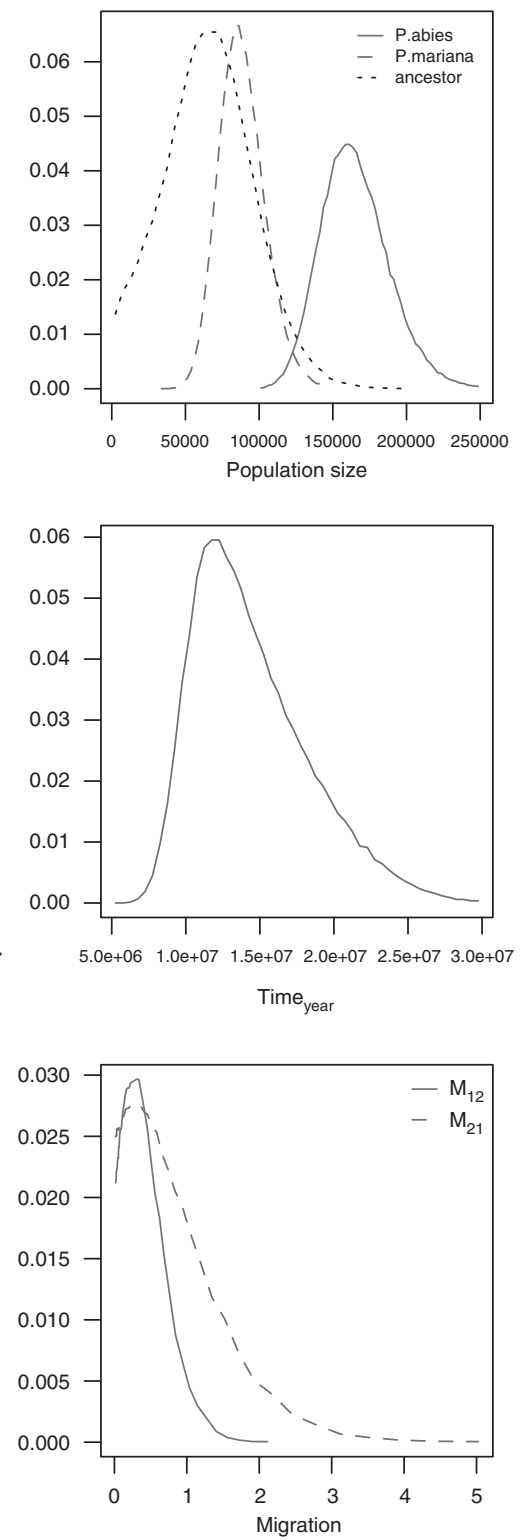

Figure 3 The smoothed marginal posterior distributions of estimated parameters under the standard Isolation-with-Migration (IM) model estimated by MIMAR $\left(\mu=1.0 \times 10^{-8}\right.$ per site per generation, 50 years per generation). (a) P. abies $\times$ P. breweriana, (b) P. abies $\times$ P. glauca and (c). P. abies $\times$ P.mariana.

the standard IM model (Figure 5). The two exceptions were $S_{\mathrm{f}}$ for $P$. abies and P. glauca, and $S_{\mathrm{f}}$ and $\pi_{2}$ for $P$. abies and P. mariana (Supplementary File S6). The poor fit of $S_{\mathrm{f}}$ $(P<0.01)$ could be because of too small number of fixed polymorphisms between $P$. abies and the two North American species (Supplementary Table S3), whereas poor fit of $\pi_{2}$ for P.abies and P. mariana $(P<0.05)$ suggests that a simple growth model may not capture every aspect of the species history.

\section{Phylogeny}

The species tree given by BEST supported the relationship inferred by the estimates of divergence time given by MIMAR (Figure 8). P. breweriana was at the basal position of the four species we analyzed, and P. abies and P. glauca clustered together.

\section{Discussion}

The main findings of the present study are the following. First, nucleotide variation was in general relatively low, considering the large distribution range of the boreal species and the notoriously high levels of heterozygosity at allozyme loci in conifer species. Nucleotide variation was also much lower in P. breweriana than in the other three species and its frequency spectrum was closer to standard neutral expectations. All three boreal species, on the other hand, had patterns similar to those observed in earlier studies of conifer species ( $P$. glauca, Bouillé and Bousquet, 2005; P. abies, Heuertz et al., 2006; and Pinus sylvestris, Pyhäjärvi et al., 2007), likely reflecting recent population growth and/or past bottleneck(s). Second, shared polymorphisms were extensive and fixed sites almost entirely confined to the pairwise comparisons 
a
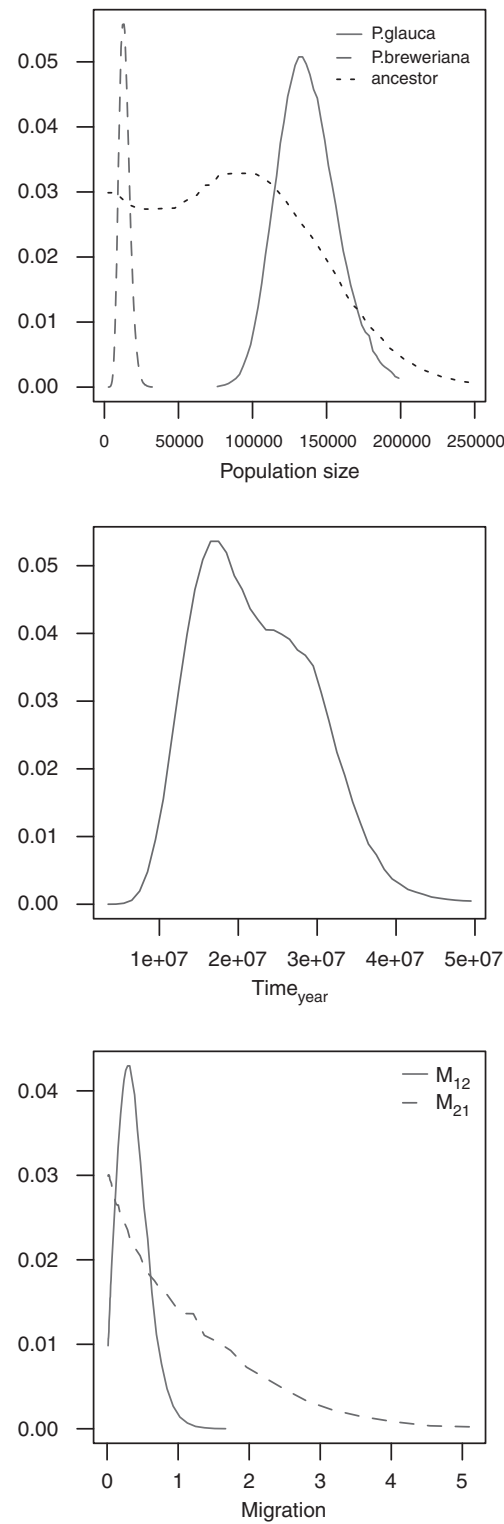

b
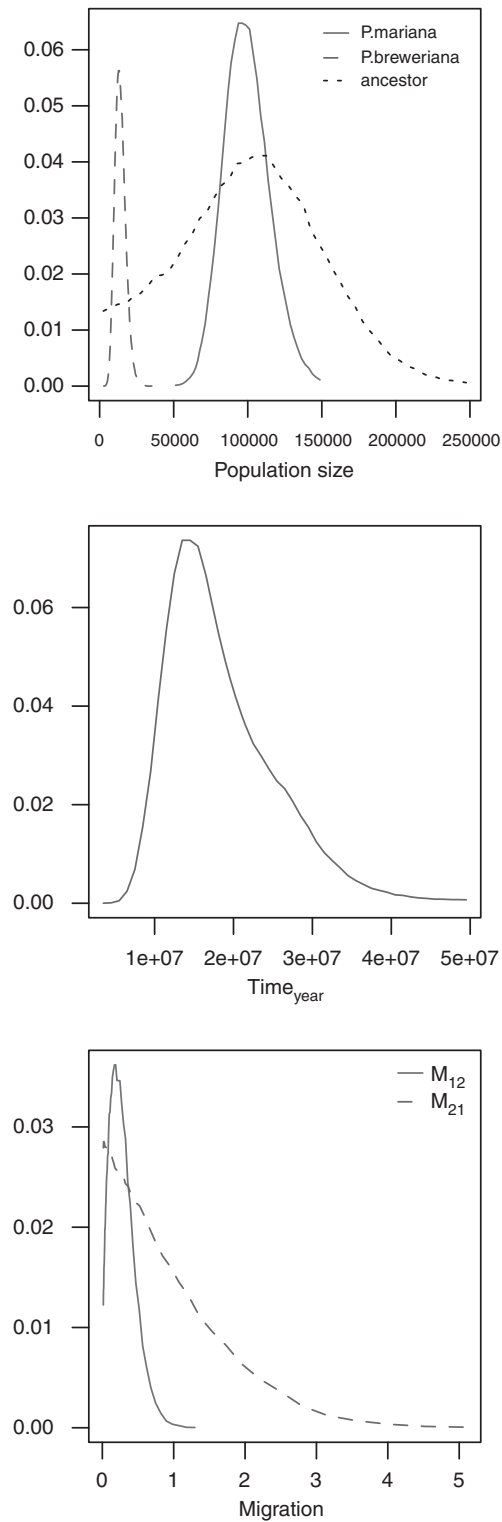

C
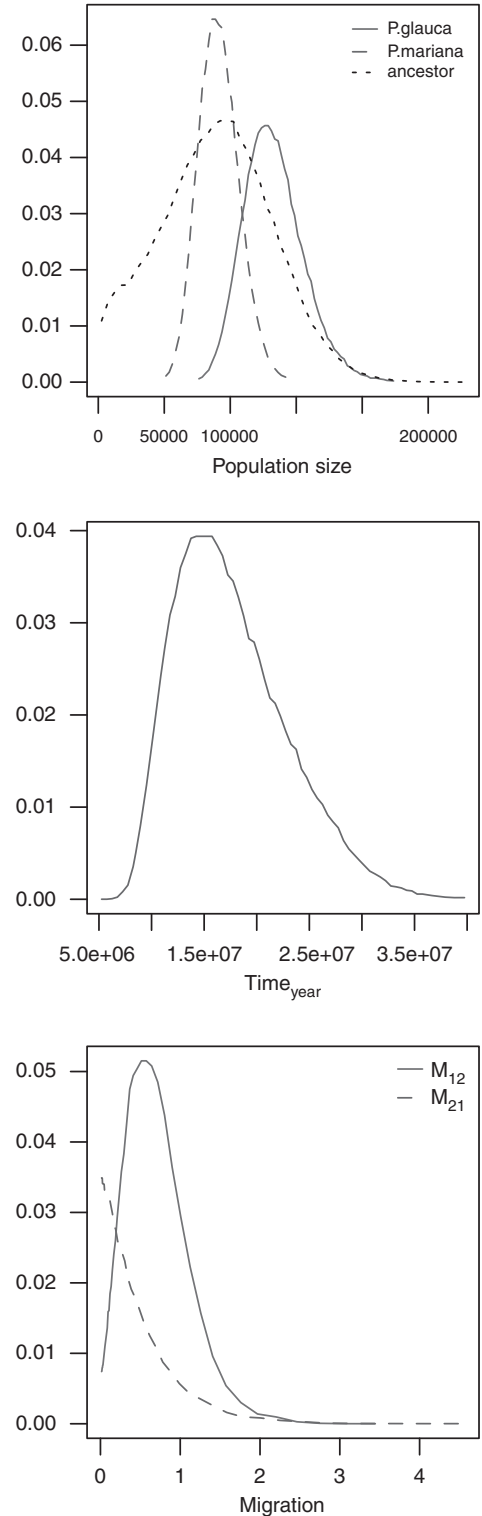

Figure 4 The smoothed marginal posterior distributions of estimated parameters under the standard Isolation-with-Migration (IM) model estimated by MIMAR $\left(\mu=1.0 \times 10^{-8}\right.$ per site per generation, 50 years per generation). (a) P. glauca $\times$ P. breweriana, (b) P. mariana $\times$ P. breweriana and (c). P. glauca $\times$ P. mariana.

involving P. breweriana. Estimates of effective populations sizes tended to be larger or of the same order of magnitude than previously published ones (Bouillé and Bousquet, 2005; Pyhäjärvi et al., 2007) except in $P$. breweriana, and divergence time much shorter than previous estimates (Bouillé and Bousquet, 2005). In particular, the divergence time between $P$. abies and the two North American boreal species P. mariana and $P$. glauca was shorter than the divergence time between the latter two, and there was even evidence for gene flow between $P$. abies and P. glauca. Among the species in this study and under all models, the effective population size of $P$. abies was the largest. This is also at variance with earlier species comparison (Bouillé and Bousquet, 2005), where $P$. abies had the smallest effective population size. This later result may simply indicate that estimates based on too few loci and too few sampled individuals are not reliable.
Polymorphism at allozyme and nucleotide levels In contrast to the similar and high levels of polymorphisms observed at allozyme loci among the four spruce species studied here (Lagercrantz and Ryman, 1990; Ledig et al., 1997 and references therein, 2005), nucleotide variation was an order of magnitude lower in $P$. breweriana than in its boreal relatives. This low value is in agreement with the fossil record and with a mean Tajima's $D$ value close to zero, both of which lent support to a scenario where $P$. breweriana retained a small population size since the Tertiary and through recent times. A similar discrepancy between levels of polymorphism at allozyme and nucleotide level has already been noted, in particular in nucleotide polymorphism surveys in P. abies (Heuertz et al., 2006) and Pinus sylvestris (Pyhäjärvi et al., 2007). In the latter case a direct comparison was done through genotyping and resequen- 

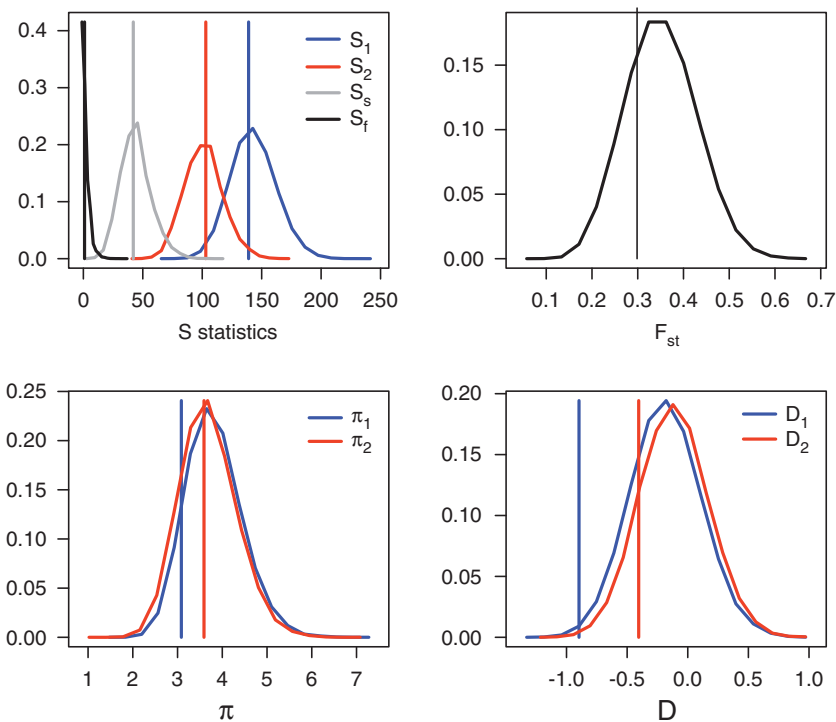

Neutral model

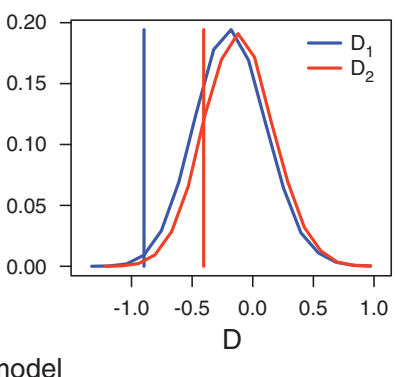

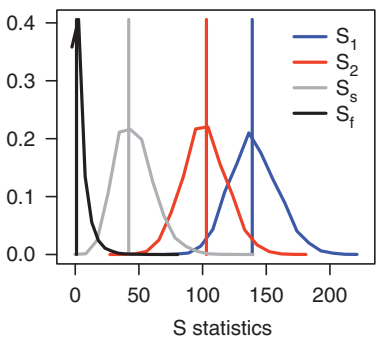

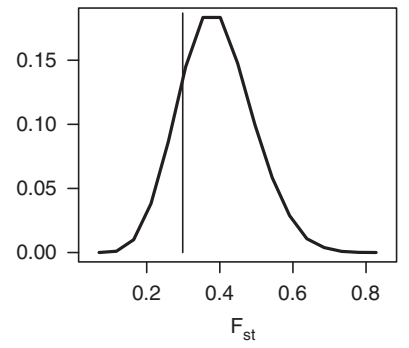

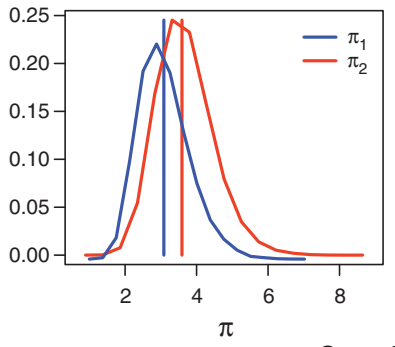

Growth model

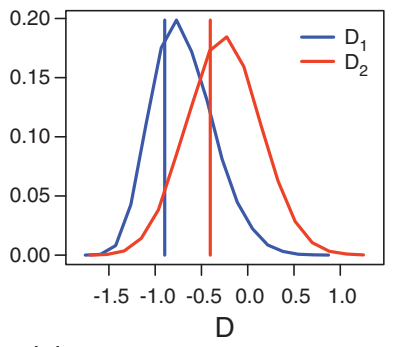

Figure 5 An example of goodness-of-fit plots from P. abies $\times$. glauca under both the neutral and growth models.

cing of six allozyme loci (Pyhäjärvi, 2008). Mutation and recombination rates were not higher at allozyme loci than at other loci. The haplotype distribution at allozymes was suggestive of recent partial sweeps or balancing selection, though more complex demographic scenarios than those tested could perhaps have also resulted in the observed pattern. As the same discrepancy seems to be observed across many species with different demographic histories, such as P. breweriana and the three boreal species studied here, selection at allozyme loci seems a more likely explanation than demographics.

\section{Speciation in spruce species and biogeographical implications}

There are a number of underlying assumptions to the IM models, in general, and to MIMAR, in particular, that may not always be met in this study, and could lead to biases in demographic estimates. First, one of the main assumptions is selective neutrality, which might be problematic when using coding sequence. None of the loci in this study show significant departure from neutrality when using Tajima's $D$ or Fay and Wu's H. A single locus, ZTL, deviated from neutral patterns when using multilocus Hudson-Kreitman-Aguadé tests. However, additional MIMAR runs excluding this locus did not alter the parameter estimates significantly (data not shown). It decreased the estimates for a small amount of gene flow between P. abies and P. breweriana, which had a peak near to zero and could be an artefact of the Bayesian implementation (Nielsen and Wakeley, 2001; Hey and Nielsen, 2004). However, the estimate of gene flow from P. glauca to P. abies was hardly influenced even though ZTL was excluded (1.12 under standard IM model and 4.32 under growth model). Second, IM models further assume a two-species system, where there is no gene flow with any other species. This is clearly not the case in this study, where at least the four included species seem to have exchanged genes with each other and with species not considered here: gene flow between P. glauca and P. engelmanii, and between $P$. mariana and $P$. rubens has been documented (for example, Perron and Bousquet, 1997; Major et al., 2005), and $P$. abies and $P$. obovata have a large hybrid zone centred around the $\mathrm{Ob}$ river (Tollefsrud et al., 2008). However, because $P$. engelmanii can be considered as a subspecies of P. glauca (Rajora and Dancik, 2000) and $P$. rubens a derivative of $P$. mariana (Perron et al., 2000), putative gene flow between them and the species studied here might have a minor effect on the level of polymorphism of the latter. The $P$. abies populations included in the present study all belong to the western part of the range, and therefore putative gene flow from $P$. obovata is likely to be limited or absent. Of course, shared polymorphisms with other spruce species exist, in particular between P. abies and other Eurasian species (our own unpublished data) and we cannot rule out that ancient gene flow from other species did inflate our estimates of the current effective population sizes.

One of the most striking results is the low divergence and the presence of unidirectional gene flow between P. glauca and P. abies. This, of course, seems surprising at first glance as both species are today found on different continents. Furthermore, it is also at variance with (i) chloroplast DNA phylogenies that place P. abies and P. glauca in distinct lineages (Ran et al., 2006), and (ii) the difficulty to cross those species (Mikkola, 1969). When $P$. glauca pollen is used to fertilize $P$. abies female cones the pollen tubes penetrate through the nucellar cap and in some cases eggs are fertilized. The embryos, however, die at different stages. It is interesting to note that the pollen tubes of $P$. abies penetrate more effectively into the nucellus of $P$. glauca than the pollen tubes in the reciprocal crosses, whereas our results would have led us to expect the opposite. On the other hand, in agreement with our results that did not detect any gene flow between these species, sterility of the cross $P$. mariana $\times P$. glauca is more strongly established and seems to be due to incompatibility: the growth of the pollen tube is halted midway through the nucellar cap or sooner. So, in summary, even if crosses between P. abies 
a
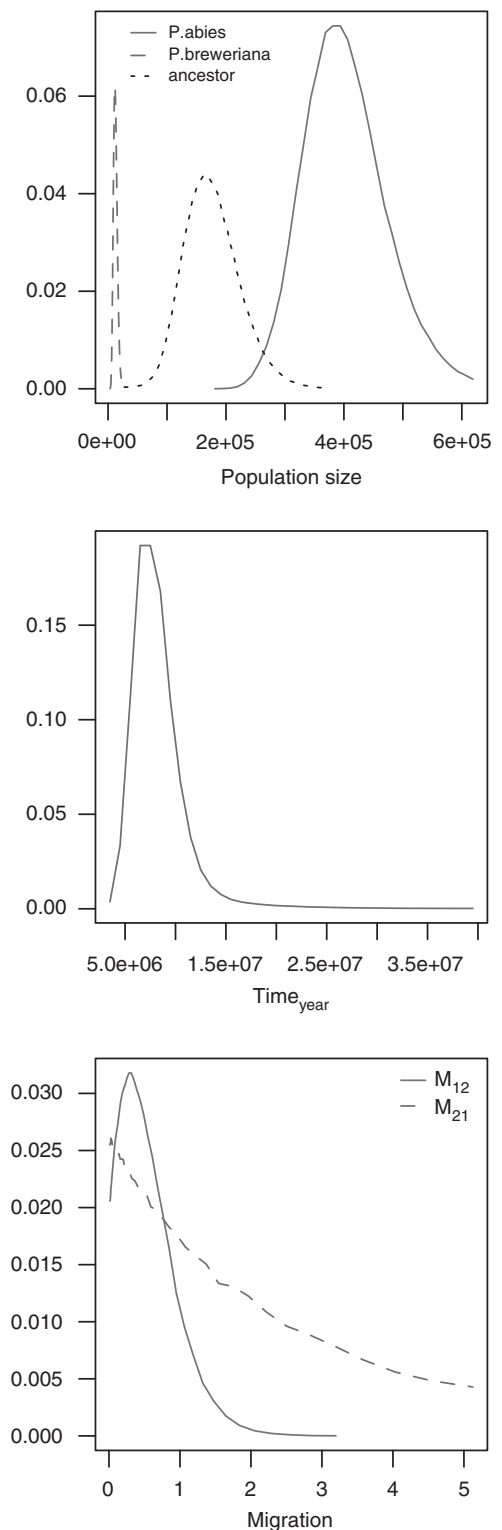

b
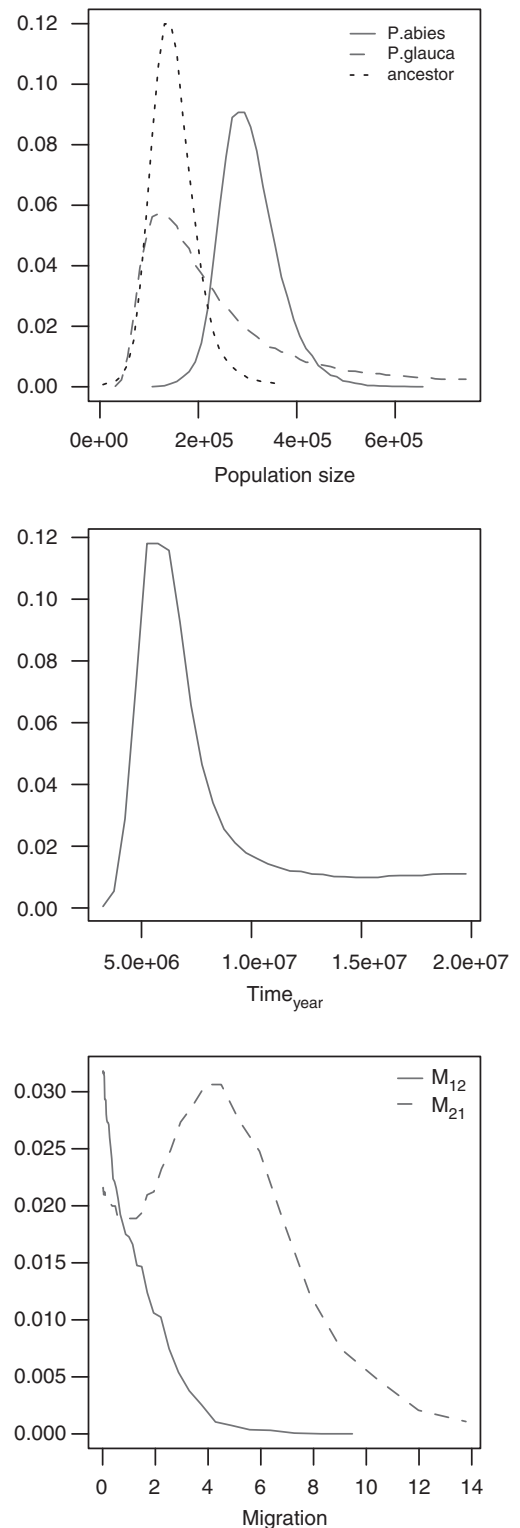

C
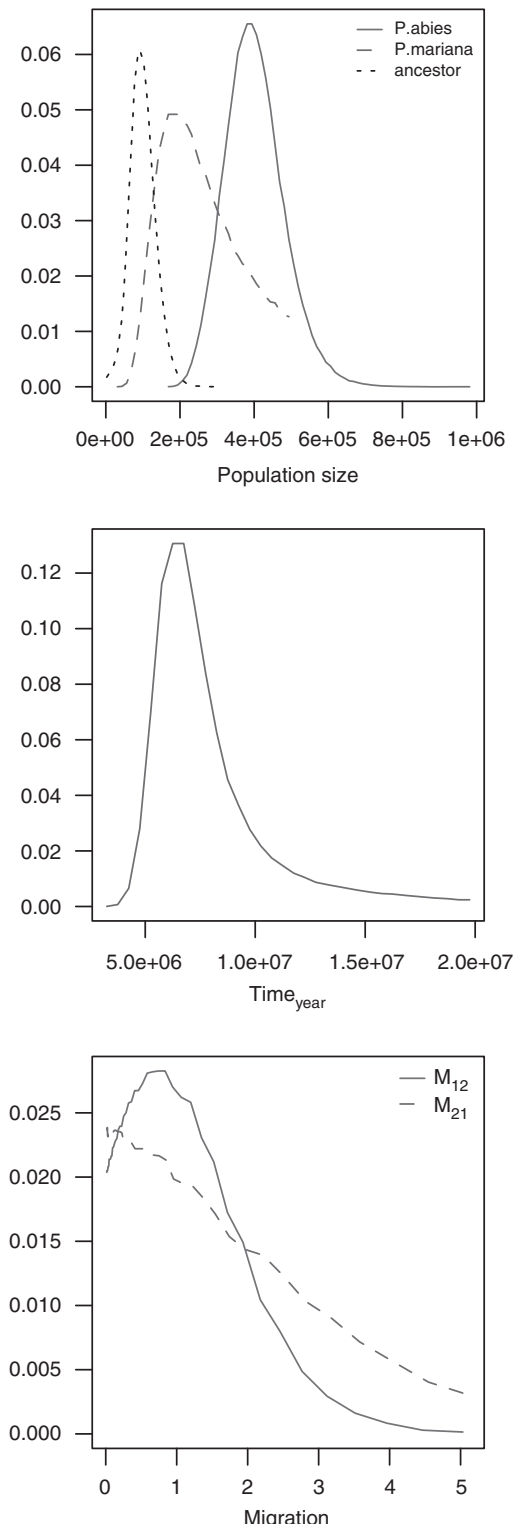

Figure 6 The smoothed marginal posterior distributions of estimated parameters under a growth model estimated by MIMAR $\left(\mu=1.0 \times 10^{-8}\right.$ per site per generation, 50 years per generation). (a) P. abies $\times$ P. breweriana, (b) P. abies $\times$ P. glauca and (c) P. abies $\times$ P. mariana.

and P. glauca are today difficult, if not impossible, they might well have been easier just after the two species diverged. Also, it is possible that $P$. obovata, which is closely related to P. abies and is geographically closer to the northern part of the range of P. glauca, acted as a bridge between the two species.

The estimates of divergence time between the four species show that $P$. breweriana separates from the others, whereas $P$. glauca was more closely related to $P$. abies than to $P$. mariana. Phylogenetic relationship based on organelle DNA could be unreliable because of higher rate of random genetic drift rate and complete linkage among loci, making mitochondrial DNA in essence equivalent to a single locus (Hudson and Coyne, 2002). Thus, we randomly picked out one individual in each species and constructed a phylogenetic tree based on multi-locus nuclear DNA using BEST programme (Liu and Pearl, 2007). The topology of the resulting tree was congruent with the relationships deduced from MIMAR estimates of divergence times. BEST, in contrast to MIMAR, assumes no recombination within locus and no migration between species. Ignoring migration amounts to assume that all shared polymorphisms are ancestral and will, therefore, lead to the overestimation of the closeness of relationship among species such as $P$. abies and $P$. glauca that have a high number of shared polymorphisms. Ignoring recombination will make trees look more star-like (Schierup and Hein, 2000). In this case none of these departures seem to have had an overwhelming effect.

\section{Conclusion}

To our knowledge this is one of the first multi-species survey of nucleotide diversity in conifers and the first study to provide estimates of IM parameters. It confirms 
b
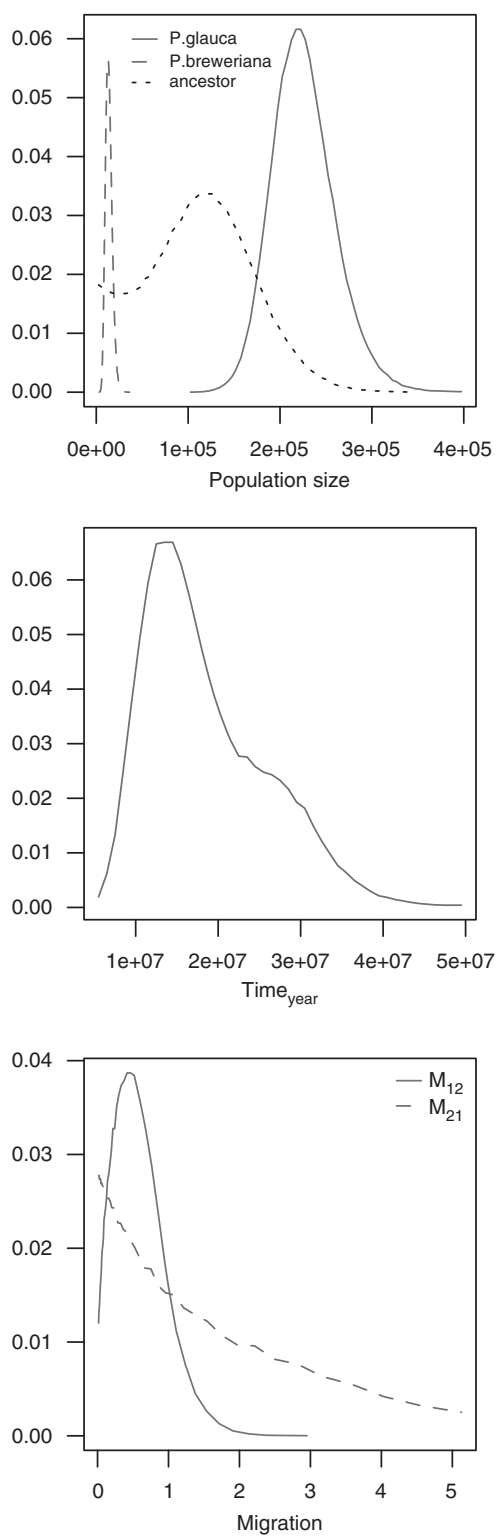

\section{C}
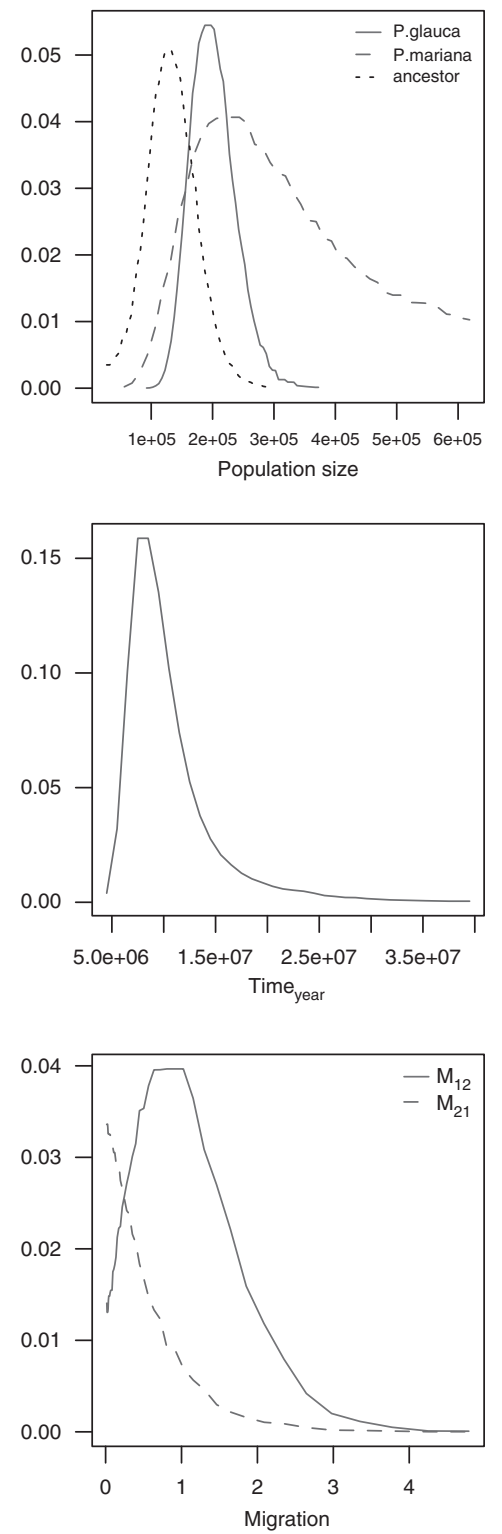

Figure 7 The smoothed marginal posterior distributions under a growth model estimated by MIMAR $\left(\mu=1.0 \times 10^{-8}\right.$ per site per generation, 50 years per generation). (a) P. glauca $\times$ P. breweriana, (b) P. mariana $\times$ P. breweriana and (c) P. glauca $\times$ P. mariana

a

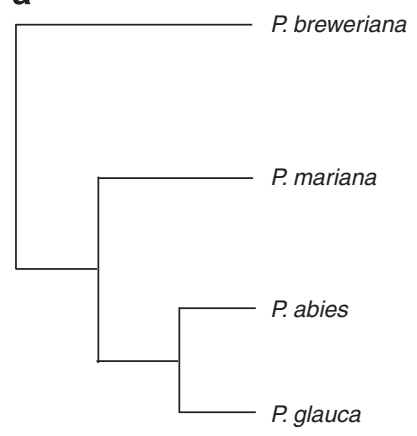

b

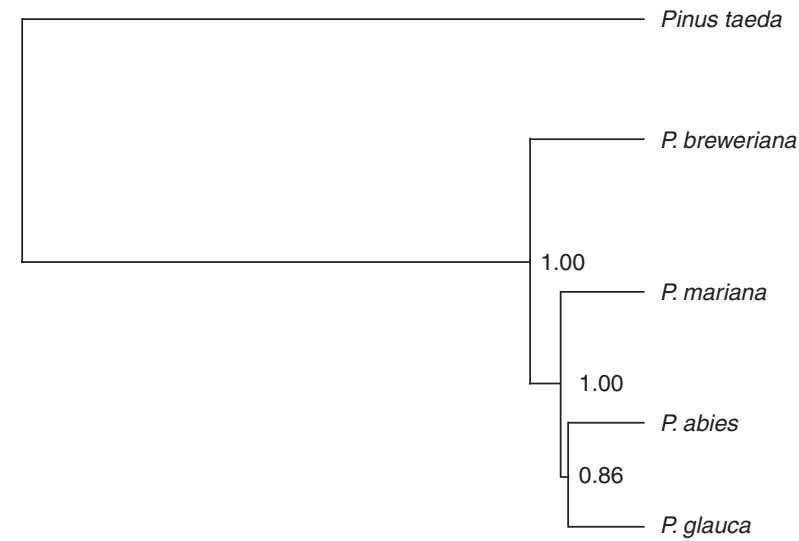

Figure 8 Phylogenetic relationships among the four studied spruce species. (a) Deduced from the divergence times estimated by MIMAR. (b) The phylogenetic tree and posterior probabilities for the topology as inferred with the programme BEST. 
the relatively low nucleotide diversity previously observed in conifers (e.g. Heuertz et al., 2006) and the extent of shared polymorphisms, and highlights the recent divergence and diversity of histories and effective population sizes among species of this important group of forest trees. The availability of well described group of species with contrasted histories and ecology will certainly prove to be very useful when assessing the relative roles played by historical contingencies and general adaptive mechanisms in trees response to environmental changes.

\section{Acknowledgements}

The research was funded by grants from the Carl Tryggers Foundation, the Philip-Sörensen Foundation and the Swedish Research Council for Environment, Agricultural Sciences and Spatial Planning (FORMAS) to Martin Lascoux. The EVOLTREE network of excellence financed Niclas Gyllenstrand. Thomas Källman received support from the Nilsson-Ehle Foundation. We thank Céline Becquet for kindly answering questions about MIMAR, and Jing Huang for help with labwork. We also want to thank Thomas Ledig and Jean Bousquet for providing us with seeds from the North American spruce species.

\section{References}

Bazin E, Glémin S, Galtier N (2006). Population size does not influence mitochondrial genetic diversity in animals. Science 312: $570-572$.

Becquet C (2007). Population genetic approaches to the study of speciation. PhD thesis, Department of Human Genetics, University of Chicago.

Becquet C, Przeworski M (2007). A new approach to estimate parameters of speciation models with application to apes. Genome Res 17: 1505-1519.

Bouillé M, Bousquet J (2005). Trans-species shared polymorphisms at orthologous nuclear gene loci among distant species in the conifer Picea (Pinaceae): Implications for the long-term maintenance of genetic diversity in trees. Am J Bot 92: 63-73.

De Vernal A, Hillaire-Marcel C (2008). Natural variability of Greenland climate, vegetation, and ice volume during the past million years. Science 320: 1622-1625.

Ewing B, Green P (1998). Base-calling of automated sequencer traces using phred. II. Error probabilities. Genome Res 8: 186-194.

Ewing B, Hillier L, Wendl MC, Green P (1998). Base-calling of automated sequencer traces using phred. I. Accuracy assessment. Genome Res 8: 175-185.

Haddrill P, Thornton KR, Charlesworth B, Andolfatto P (2005). Multilocus patterns of nucleotide variability and the demographic and selection history of Drosophila melanogaster populations. Genome Res 15: 790-799.

Heuertz M, De Paoli E, Källman T, Larsson H, Jurman I, Morgante M et al. (2006). Multilocus patterns of nucleotide diversity, linkage disequilibrium and demographic history of Norway Spruce [Picea abies (L.) Karst]. Genetics 174: 2095-2105.

Hey J, Nielsen R (2004). Multilocus methods for estimating population sizes, migration rates and divergence time, with applications to the divergence of Drosophila pseudoobscura and D. persimilis. Genetics 167: 747-760.

Hudson RR, Coyne JA (2002). Mathematical consequences of the genealogical species concept. Evolution 56: $1557-1565$
Gordon D, Abajian C, Green P (1998). Consed: A graphical tool for sequence finishing. Genome Res 8: 195-202.

Ingvarsson PK (2008). Multilocus patterns of nucleotide polymorphism and the demographic history of Populus tremula. Genetics 180: 329-340.

Innan H, Kim Y (2008). Detecting local adaptation using the joint sampling of polymorphism data in the parental and derived populations. Genetics 179: 1713-1719.

Lagercrantz U, Ryman N (1990). Genetic structure of Norway spruce (Picea abies): Concordance of morphological and allozymic variation. Evolution 44: 38-53.

Ledig FT, Hodgskiss P, Johnson D (2005). Genetic diversity, genetic structure, and mating system of Brewer spruce (Pinaceae), a relict of the Arcto-Tertiary forest. Am J Bot 92: 1975-1986.

Ledig FT, Jacob-Cervantes V, Hodgkiss PD, Equiluz-Piedra T (1997). Recent evolution and divergence among populations of a rare Mexican endemic, Chihuahua spruce, following Holocene climatic warming. Evolution 51: 1815-1827.

Liu L, Pearl DK (2007). Species trees from gene tree: Reconstructing Bayesian posterior distribution of a species phylogeny using estimated gene tree distributions. Syst Biol 56: 504-514.

Major JE, Mosseler A, Johnsen KH, Rajora BP, Barci DC, Kim $\mathrm{KH}$ et al. (2005). Reproductive barriers and hybridity in two spruces, Picea rubens, Picea mariana, sympatric in eastern North America. Can J Bot 83: 163-175.

McVean G, Awadalla P, Fearnhead P (2002). A coalescent-based method for detecting and estimating recombination from gene sequences. Genetics 160: 1231-1241.

Mikkola L (1969). Observations on interspecific sterility in Picea. Ann Bot Fennici 6: 285-339.

Nielsen R, Wakeley J (2001). Distinguishing migration from isolation: A Markov chain Monte Carlo approach. Genetics 158: $885-896$.

Patterson N, Richter DJ, Gnerre S, Lander ES, Reich D (2006). Genetic evidence for complex speciation of humans and chimpanzee. Nature 441: 1103-1108.

Perron M, Bousquet J (1997). Natural hybridization between black spruce and red spruce. Mol Ecol 6: 725-734.

Perron M, Perry DJ, Andalo C, Bousquet J (2000). Evidence from sequence-tagged-site markers of a recent progenitorderivative species pair in conifers. Proc Natl Acad Sci USA 97: 11331-11336.

Pyhäjärvi T (2008). Roles of demography and natural selection in molecular evolution of trees, focus on Pinus sylvestris. $\mathrm{PhD}$ thesis, University of Oulu, Finland.

Pyhäjärvi T, García-Gil MR, Knürr T, Mikkonen M, Wachowiak W, Savolainen O (2007). Demographic history has influenced nucleotide diversity in European Pinus sylvestris populations. Genetics 177: 1713-1724.

Rajora O, Dancik BP (2000). Population genetic variation, structure, and evolution in Engelmann spruce, white spruce, and their natural hybrid complex in Alberta. Can J Bot $\mathbf{7 8}$ 768-780.

Ran JH, Wei XX, Wang XQ (2006). Molecular phylogeny and biogeography of Picea (Pinaceae): Implications for phylogeographical studies using cytoplasmic haplotypes. Mol Phylogenet Evol 41: 405-419.

Ronquist F, Huelsenbeck JP (2003). MRBAYES: Bayesian phylogenetic inference under mixed models. Bioinformatics 19: $1572-1574$.

Rozas J, Sánchez-DelBarrio JC, Messeguer X, Rozas R (2003). DnaSP, DNA polymorphism analyses by the coalescent and other methods. Bioinformatics 19: 2496-2497.

Schierup MH, Hein J (2000). Consequences of recombination on traditional phylogenetic analysis. Genetics 156: 879-891.

Slotte T, Huang H, Lascoux M, Ceplitis A (2008). Polyploid speciation did not confer instant reproductive isolation in Capsella (Brassicaceae). Mol Biol Evol 25: 1472-1481. 
Thornton K (2003). Libsequence: A C++ class library for evolutionary genetic analysis. Bioinformatics 19: 2325-2327.

Tollefsrud MM, Kissling R, Gugerli F, Johnsen O, Skrøppa T, Cheddadi $\mathrm{R}$ et al. (2008). Genetic consequences of glacial survival and postglacial colonization in Norway spruce: Combined analysis of mitochondrial DNA and fossil pollen. Mol Ecol 17: 4134-4150.

Wakeley J (2008). Coalescent theory-An introduction. Roberts and Company Publishers.
Wakeley J, Hey J (1997). Estimating ancestral population parameters. Genetics 145: 847-855.

Wright SJ, Charlesworth B (2004). The HKA test revisited: A maximum likelihood ratio test of the standard neutral model. Genetics 168: 1071-1076.

Zhai W, Nielsen R, Slatkin M (2009). An investigation of the statistical power of neutrality tests based on comparative and population genetic data. Mol Biol Evol 26: 273-283.

Supplementary Information accompanies the paper on Heredity website (http://www.nature.com/hdy) 